\title{
GW23-e0894 CLINICAL APPLIED RESEARCH OF PERCUTANEOUS BALLOON PULMONARY VALVE FOR CONGENITAL PULMONARY VALVE STENOSIS
}

doi:10.1136/heartjnl-2012-302920p.5

${ }^{1}$ Xie Dongming, ${ }^{1}$ xie dongyang, ${ }^{1}$ yan yihong, ${ }^{2}$ Xie Dongming. ${ }^{1}$ Affiliated Hospital of Gannan Medical College; ${ }^{2}$ Affiliated Hospital of Gannan Medical College

Objectives To evaluate the clinical efficacy of percutaneous balloon pulmonary valvuloplasty (PBPV) for pulmonary valve stenosis (PS) Methods 20 patients, through clinical, ECG, chest X-ray and cardiac ultrasound, were diagnosis as pure PS. Before PBPV, determination PS's type and valve annulus size, to select balloon diameter larger than its about 20-40\%. After balloon expansion instantly measured pulmonary pressure gradient, and observed the pulmonary valve open, ejection, pressure gradient, tricuspid and pulmonary valve regurgitation after PBPV.

Results All 20 patients were successful with the balloon dilation, pressure gradient decreased $63.65 \%(\mathrm{p}<0.01), 1$ patients had mild pulmonary valve regurgitation, and 1 case in intraoperative occurred sinus bradycardia and disappeared by using atropine, no any serious complications.

Conclusions PBPV treatment for pulmonary valve stenosis with a high success rate, little trauma and few complications, and could be the first choice for PS treatment. 